BIOMEDICA

Vol. 5, Nos. 3 y $4-1985$

\title{
CUATROCIENTOS SETENTA Y SIETE CASOS DE URETRITIS MASCULINA
}

\author{
FEDERICO DIAZ GONZALEZ.*
}

\begin{abstract}
En el año de 1985 se estudiaron, desde el punto de vista del laboratorio, 477 casos de uretritis masculina; 49 de ellos $(10.3 \%)$ fueron de uretritis gonocócica (UG) y 428 (89.7\%) de uretritis no gonocócica (UNG). Estos resultados concuerdan con los obtenidos en series previas; se discute la importancia de la UNG y se plantea la posibilidad de que muchos de los casos de esta entidad sean de la modalidad postgonocócica (UPG).
\end{abstract}

\section{INTRODUCCION}

La uretritis masculina, que se manifiesta por secreción uretral y disuria, es un motivo de consulta frecuente; puede ser causada por Neisseria gonorrhoeae en cuyo caso se habla de uretritis gonocócica (UG) o por otros agentes y entonces se alude a ella como uretritis no gonocócica (UNG); el principal microorganismo entre los causantes de UNG es la Chlamydia trachomatis (1).

En tabulaciones previas de series de pacientes con uretritis $(2,3,4,5)$ hemos encontrado un abierto predominio de la UNG, en pacientes de la práctica privada o de entidades oficiales; en contraste fue mucho más frecuente la UG en una corta serie de pacientes que consultaron al Centro de Salud No. 25 de la ciudad de Medellín (6); estos últimos son de un nivel socioeconómico más bajo que los primeros pero la discrepancia en la frecuencia de UG y UNG también podría ser debida al hecho de que en la serie del Centro de Salud se exigió que no hubiera antecedente de antibioterapia en los últimos seis meses.

\section{MATERIALES Y METODOS}

Los pacientes fueron remitidos con base en la clínica e incluídos en el estudio sólo si cumplían los criterios de laboratorio que permiten sustentar un diagnóstico de uretritis (7); la diferenciación entre UG y UNG se estableció con base en la coloración de Gram de la secreción uretral o del sedimento de la primera porción de orina matinal; su confiabilidad, determinada en estudios previos (4), supera el $90 \%$. En esta serie no se pretendió establecer correlaciones entre la etiología y otras variables.

\section{RESULTADOS}

Cuarenta y nueva pacientes $(10.3 \%)$ tenían UG y $428(89.7 \%)$ UNG.

\section{DISCUSION}

Es obvio que en pacientes de un determinado estrato socio económico predomina la UNG; sin embargo hay que tener presente el hecho de que en ésta y en varias series anteriores $(3,4,5)$ se estudiaron los pacientes independientemente de su historia previa de antibioterapia; ello plantea la posibilidad, que puede ser objeto de investigación en el futuro, de que una proporción apreciable de los casos de UNG sean de la modalidad postgonocócica (UPG); independientemente de esta posibilidad parece claro que el tratamiento de elección en esta población es una de las tetraciclinas.

*Profesor Titular, Facultad de Medicina, Departamento de Microbiología y Parasitología, Universidad de Antioquia. 


\section{SUMMARY}

During 1985 the author studied 477 men with clinical and laboratory evidence of urethritis; $49(10.3 \%)$ were diagnosed as gonococcal urethritis while $428(89.7 \%)$ had the nongonococcal form; these findings agree with those from previous series; the fact that the patients were included irrespectively of the history of previous antimicrobial therapy suggests that a proportion of the nongonococcal cases could be of the postgonococcal modality.

\section{AGRADECIMIENTO}

A los médicos que nos dispensaron su confianza; a las bacteriólogas María Isabel Vélez, Olga Lucía Jiménez y Silvia Inés Ochoa por su invaluable dedicación.

\section{BIBLIOGRAFIA}

1. Oriel, J.D. Genital infection in men. Brit Med Bull, 1983, 39 (2): 133-137.
2. Diaz, F. Uretritis masculina gonocócica y no gonocócica. Papel del laboratorio clínico en su diagnóstico microbiológico. Biomédica, 1982, 2 (4) : 172-176.

3. Díaz, F. Uretritis masculina. Estudio prospectivo de 254 casos. Biomédica, 1983, 3 (1-2): 5-9.

4. Diaz, F. Uretritis masculina. Aspectos clínicos, microbiológicos, epidemiológicos y terapéutticos en 299 pacientes. Biomédica, 1984, 4 (2): 59-66.

5. Díaz, F., Jaramillo, G., Vélez, M.I., Jiménez, O.L., Ochoa, S.I. Quinientos diez casos de uretritis masculina. Temas Microbiológicos, $1985,6(1): 1-6$.

6. Vásquez, M.E., Díaz, F. Etiología de la uretritis masculina. Estudio en el Centro de Salud No. 25 de la ciudad de Medellín. Medicina UPB, 1986. En prensa.

7. Desai, K., Robson, H.G. Comparison of the Gram-stained smear and first-voided urine sediment in the diagnosis of nongonococcal urethritis. Sex Transm Dis, 1982, 9 (1): 21-25. 